\title{
Molecular genetics, genetic testing, novel genome sequencing technologies
}

\author{
Gerard Pals* \\ Department of Clinical Genetics, VU University Medical Center Amsterdam, \\ Netherlands
}

DOI: http://dx.doi.org/10.19106/JMedScieSup004804201609

\begin{abstract}
With the advance of genomic technologies, we are now able to detect genetic variations in patients with high accuracy involving whole genome scale, as the procedure is relatively cost-effective. This offers an opportunity to fundamentally alter medical practice and insurance policy. Although clinicians, scientists, and health policy makers still have to deal with how to interpret and handle the results, since sometimes it comes with ambiguity and uncertainty, recent advances especially in the Western world have integrated genetic tests and molecular genetic analysis for clinical management of patients. In this article, we will discuss and review the range of methods currently used in clinical setting, as well as potential emerging methods in clinical molecular genetics diagnostics. Advantages and disadvantages of each methods will be carefully discussed, especially application in regions of the world that have more limited access for molecular genetic tests including next generation sequencing. Outline of implementation challenges for molecular genetic tests both in terms of health economics and clinical management will also be discussed.
\end{abstract}

Keywords: molecular genetics, diagnostics, next generation sequencing 